\title{
Uso problemático de Internet y variables psicológicas o físicas en estudiantes universitarios
}

\section{Problematic Internet Use and Psychological or Physical Variables in University Students}

Francisco Javier Hinojo-Lucena (*) https://orcid.org/0000-0002-9507-4058

Inmaculada Aznar-Díaz (*) https://orcid.org/0000-0002-0018-1150

Juan Manuel Trujillo-Torres (*) https://orcid.org/0000-0002-2761-3523

José María Romero-Rodríguez (*) https://orcid.org/0000-0002-9284-8919

(*) Universidad de Granada

(Recibido: 4 de julio de 2019; Aceptado para su publicación: 12 de noviembre de 2019)

Cómo citar: Hinojo-Lucena, F. J., Aznar-Díaz, I., Trujillo-Torres, J. M. y Romero-Rodríguez, J. M. (2021). Uso problemático de Internet y variables psicológicas o física en estudiantes universitarios. Revista Electrónica de Investigación Educativa, 23, e13, 1-17. https://doi.org/10.24320/redie.2021.23.e13.3167

\section{Resumen}

El Uso roblemático de Internet (UPI) está siendo habitual entre los estudiantes universitarios. En este trabajo se propuso identificar las variables psicológicas y físicas asociadas al UPI y analizar su influencia en dichas variables. Se empleó una metodología de revisión sistemática con metanálisis, en las bases de datos Web of Science y Scopus. En la revisión se identificaron 51 estudios que incluyeron una muestra total de 136760 sujetos. En cambio, para el metanálisis se identificaron 12 estudios. Los resultados mostraron que las principales variables psicológicas y físicas asociadas al UPI son la falta de autocontrol, ansiedad, baja autoestima, baja actividad física y baja autoeficacia académica. Sin embargo, el metanálisis recogió que sólo el UPI, donde se obtuvieron diferencias estadísticamente significativas, fue predictor de la ansiedad y baja autoestima. Por último, se discuten las implicaciones prácticas del trabajo y la necesidad de prevenir y educar en el buen uso de la tecnología.

Palabras clave: internet, estudiante universitario, comportamiento, adicción, psicología

\begin{abstract}
Problematic Internet Use (PIU) is becoming common among university students. This research set out to identify the psychological and physical variables associated with PIU and examine the influence of PIU on these variables. A systematic review and meta-analysis were conducted in the Web of Science and Scopus databases. The review found 51 studies with a total sample size of 136,760 subjects, while 12 studies were identified for the meta-analysis. The results showed that the main psychological and physical variables associated with PIU are a lack of self-control, anxiety, low self-esteem, low levels of physical activity, and low academic self-efficacy. However, the meta-analysis found that only PIU - for which statistically significant differences were observed - was a predictor of anxiety and low self-esteem. Lastly, this paper discusses the practical implications of this research and the need for preventive action and education on the proper use of technology.
\end{abstract}




\section{Introducción}

El avance de las Tecnologías de la Información y Comunicación (TIC) y la llegada de Internet han convertido a la sociedad del siglo XXI en una sociedad digitalizada (Martínez y Rodríguez-García, 2018). Esta sociedad se caracteriza por la facilidad de acceso a la información, el uso cotidiano de la tecnología y la digitalización de una gran parte de trámites con la administración pública.

A raíz de ello, la repercusión tecnológica ha influido en el tiempo de conexión a Internet por parte de la población estudiantil (Ruiz-Palmero et al., 2016). En España destacan dos importantes hitos acontecidos en los últimos tiempos: 1) presenta la mayor tasa de smartphones por habitante del mundo (Europa Press, 2017); y 2) el 93\% de la población española está conectada a Internet (We are social, 2019). Estos dos acontecimientos sitúan a España como un país en riesgo de adicción a Internet. Tal es así, que ya posee la mayor tasa de uso problemático de Internet (UPI) de Europa (Díaz-Aguado et al., 2018).

Un factor condicionante en la población juvenil es la falta de control y supervisión de los padres sobre las actividades que realizan sus hijos en Internet, lo que aumenta la probabilidad de encontrarse con diferentes peligros en la red (Symons et al., 2019). En concreto, los mayores peligros se relacionan con el grooming (engaño al menor por parte de un adulto) y el sexting (compartir imágenes con contenido sexual) (Machimbarrena et al., 2018). Además de acrecentar la probabilidad de sufrir cyberbullying si se realiza un uso abusivo de Internet (Giménez et al., 2017; Kopecký y Szotkowski, 2017).

Por otra parte, algunos estudios indican que pasar tiempo excesivo en Internet es un predictor del UPI (Tabachuk et al., 2018), y la población en riesgo la integran adolescentes y estudiantes universitarios (García-Oliva et al., 2017), por lo que la adicción a Internet es un problema que afecta principalmente a los más jóvenes y se asocia a las de tipo comportamental. Así pues, provoca en el usuario estímulos internos y pérdida de control de sus acciones, en este caso relacionadas con el tiempo que pasa en la red.

La problemática sobre el UPI no es un fenómeno aislado en el contexto español, se han detectado tasas de prevalencia alarmantes de adicción a Internet en todo el mundo (Throuvala et al., 2019). De modo que la globalización auspiciada por la tecnología ha influido principalmente en los países desarrollados, donde han surgido enfermedades como la adicción tecnológica (Varo et al., 2019), una problemática ya a escala mundial.

Una de las principales consecuencias de un UPI es el desarrollo de síntomas psicosomáticos (Çam y Nur, 2015; Cao et al., 2011), los cuales refieren a patologías que afectan la salud física de la persona, las cuales son causadas principalmente por factores mentales. Con base en ello, se ha vinculado el UPI con bajas relaciones sociales en adolescentes (Zegarra y Cuba, 2017), bajo rendimiento académico (Ramos-Galarza et al., 2017), malestar físico (Castro et al., 2018) y la influencia en la estabilidad emocional (Koronczai et al., 2019).

El interés por el UPI se ha reflejado en los últimos tiempos con la cantidad de literatura científica publicada sobre el tópico. Estudios previos de revisión sistemática y metanálisis han abordado la temática desde distintos enfoques: Rojas-Jaras et al. (2018) realizaron una revisión sistemática sobre la adicción a Internet y el uso de redes sociales en adolescentes, donde revisaron 126 artículos científicos. Entre los resultados destaca el uso excesivo de Internet en la población adolescente y la detección de diferentes efectos negativos asociados al UPI, como: alteraciones en el sueño, bajo rendimiento académico, depresión, baja autoestima y consumo de sustancias. Por otro lado, Lei et al. (2018) analizaron 76 documentos para determinar la relación entre el apoyo social y la adicción a Internet en China. Los datos recogieron la correlación negativa entre la adicción a Internet y el apoyo social, y Lei et al. (2019) revisaron 70 documentos para determinar si el UPI estaba relacionado con el bienestar subjetivo, la satisfacción con la vida, las emociones positivas o las emociones negativas, el metanálisis confirmó que los estudiantes que registraron un excesivo de Internet tenían más emociones negativas, menos bienestar subjetivo, menos satisfacción con la vida y menos emociones positivas.

Por tanto, nos encontramos con una temática en pleno auge debido a la necesidad social de detectar qué tipo de consecuencias está teniendo la realización de un UPI en la población. Así pues, se propusieron 
como objetivos: 1) identificar las variables psicológicas y físicas asociadas al uso problemático de Internet a partir de la revisión sistemática de la literatura; y 2) analizar la influencia del uso problemático de Internet en las variable psicológicas o físicas que permitan la confección de un metanálisis. En consideración, se establecieron como interrogantes de investigación: ¿Cuál es la población que presenta una mayor tasa de UPI? ¿Cuáles son las variables psicológicas y físicas asociados al UPI? ¿Es significativa la influencia del UPI en el desarrollo de variables psicológicas o físicas entre la población control y con UPI?

\section{Método}

Se utilizó una metodología de revisión sistemática con metanálisis (Sánchez-Meca, 2010). Para dotar de rigurosidad la revisión, se siguieron los criterios de calidad de la declaración PRISMA (Moher et al., 2009).

Las variables de análisis de la revisión sistemática se establecieron conforme a los objetivos del estudio; éstas fueron: población, países, variables psicológicas y físicas asociadas al UPI e instrumentos de recogida de datos utilizados.

El metanálisis se realizó por cada una de las variables psicológicas y físicas detectadas, excepto con aquellos en los que los estudios no recogieron los datos necesarios para su confección.

\subsection{Procedimiento de obtención de la muestra}

La búsqueda se realizó a través del establecimiento de los descriptores que definieron los tópicos del estudio. En un primer momento se aplicó la ecuación de búsqueda ("Problematic Internet use" OR "Internet addiction") para identificar los variables psicológicas y físicas asociadas a la adicción a Internet. A partir de su aplicación se realizó un conteo de documentos, teniendo en consideración que la variable psicológica o física se repitiera en cinco o más estudios. Una vez identificadas las variables psicológicas y físicas (falta de autocontrol, ansiedad, autoestima, baja actividad física y baja autoeficacia académica) se realizaron búsquedas con cada descriptor: ("Problematic Internet use" OR "Internet addiction") AND Anxiety; ("Problematic Internet use" OR "Internet addiction") AND Self-control; ("Problematic Internet use" OR "Internet addiction") AND Self-esteem; ("Problematic Internet use" OR "Internet addiction") AND Physical activity; ("Problematic Internet use" OR "Internet addiction") AND ("Academic self-efficacy" OR "Academic performance").

Las bases de datos seleccionadas fueron Web of Science (WOS) y Scopus, ya que son las dos bases de datos que presentan los índices de impacto más importantes y reconocidos por la comunidad internacional (Sola et al., 2019). Así pues, las diferentes ecuaciones de búsqueda se aplicaron en los motores de búsqueda de ambas bases de datos. Tras su aplicación se introdujeron distintos criterios de inclusión y exclusión para proceder al refinado (Hinojo et al., 2019).

Los criterios de inclusión fueron: 1) artículos de revista; 2) publicado en abierto y disponible para su consulta; 3) estudios empíricos; 4) asociación del UPI con una variable psicológica o física. Mientras que los criterios de exclusión se relacionaron con: 1) actas de congresos, capítulos de libro, libros u otro tipo de publicaciones no revisadas por pares; 2) acceso restringido a la publicación; 3) estudios teóricos o revisiones; 4) los estudios no vinculan el UPI a ninguna variable psicológica o física.

Con la finalidad de evitar el sesgo de selección de los estudios, dos investigadores independientes revisaron la literatura aplicando los mismos descriptores y criterios de inclusión y exclusión. El grado de acuerdo fue del 99\%. Las discrepancias fueron resueltas con la inclusión del 100\% de la literatura extraída por cada investigador. 


\subsection{Recopilación de datos}

El refinado de la literatura fue realizado con base en la declaración PRISMA (Moher et al., 2009) (figura 1). Se establecieron cuatro fases de filtrado. La fase de identificación consistió en la búsqueda inicial de referencias en las dos bases de datos y en la identificación de documentos en otras fuentes. Posteriormente, en la fase de cribado se emplearon los criterios de inclusión (1, 2 y 3) y los criterios de exclusión (1, 2 y 3). Enseguida, la fase de idoneidad refinó la búsqueda aplicando los criterios de inclusión (4 y 5) y de exclusión (4 y 5). Por último, la fase de inclusión recogió los documentos que conformaron la revisión sistemática $(n=51)$ y el metanálisis $(n=12)$. La búsqueda se realizó el 10 de marzo de 2019 y se recogieron todos los documentos publicados hasta la fecha.

Figura 1. Diagrama de flujo de acuerdo a la declaración PRISMA

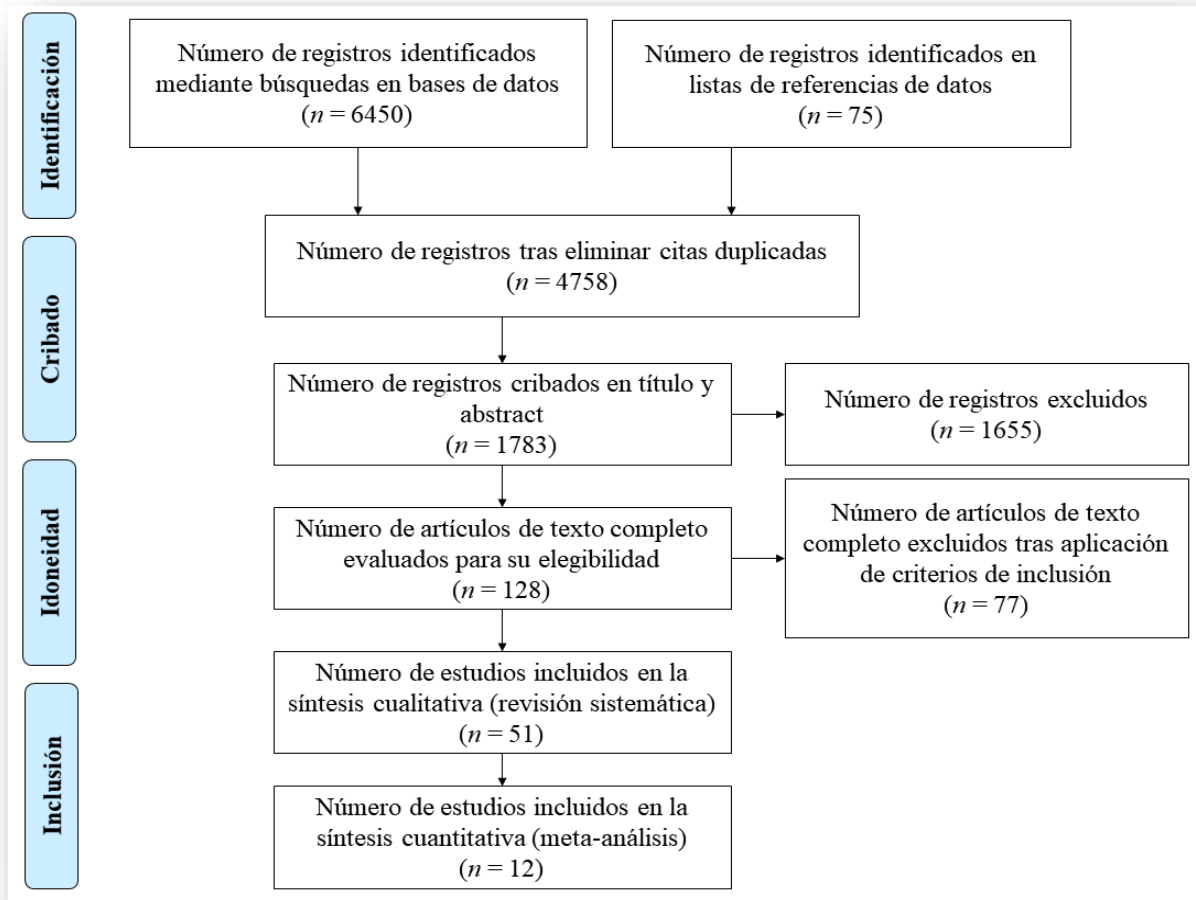

\subsection{Análisis de datos}

El análisis de datos se realizó por medio de la lectura pormenorizada de los documentos para la extracción de las variables de interés para el estudio (población, países, variables psicológicas o físicas e instrumentos de recogida de datos).

No obstante, para la confección de los múltiples metanálisis sólo se incluyeron los artículos que contuvieran los datos referentes a la media y desviación típica de los grupos de control y los grupos con UPI. Sin embargo, hubo una excepción en la variable "baja actividad física". A este respecto, se realizó un metanálisis de metodología debido a la característica de los estudios analizados, donde sólo se contemplaba el número total de participantes activos e inactivos sin recurrir a datos estadísticodescriptivos.

Los datos del metanálisis se analizaron con el software Review Manager, versión 5.3. El intervalo de confianza se estableció en 95\%. 


\section{Resultados}

Los 51 estudios analizados recogieron una muestra total de 136.760 sujetos, la cual osciló entre 60 y 70.696 participantes $(M=2.681,56 ; S D=10.025,54)$. En relación con la población objeto de estudio (ver tabla 1), la mayoría fueron estudiantes universitarios (45.09\%), seguido de adolescentes (29.41\%) y adultos (27.45\%). A su vez, la edad media se situó entre los 12 y 42 años $(M=21,19 ; S D=6.34)$. Mientras que la distribución de países quedó configurada de la siguiente manera: Turquía (33.33\%); Estados Unidos (EEUU) (15.68\%); Corea del Sur (9.80\%); España (7.84\%); Reino Unido (UK) (5.88\%); China (5.88\%); Japón (3.92\%); Italia (3.92\%); Australia (3.92\%); Singapur (1.96\%); Polonia (1.96\%); Sudáfrica (1.96\%); Portugal (1.96\%); Francia (1.96\%). 
Tabla 1. Características de la población

\begin{tabular}{|c|c|c|c|c|c|c|}
\hline \multirow[b]{2}{*}{ Referencia } & \multicolumn{3}{|c|}{ Sector poblacional } & \multirow[b]{2}{*}{$n$} & \multirow[b]{2}{*}{$\begin{array}{c}\text { Edad } \\
\text { (M) }\end{array}$} & \multirow[b]{2}{*}{ País } \\
\hline & $\begin{array}{c}\text { Adoles- } \\
\text { centes }\end{array}$ & Adultos & $\begin{array}{c}\text { Estudiantes } \\
\text { universitarios }\end{array}$ & & & \\
\hline Baloğlu et al. (2018) & & & $\mathrm{X}$ & 505 & 20.34 & Turquía \\
\hline Bernal-Ruiz et al. (2017) & $x$ & & $x$ & 310 & 18.25 & España \\
\hline Bozkurt et al. (2013) & $x$ & & & 60 & 13.38 & Turquía \\
\hline Cao et al. (2011) & $x$ & & & 17599 & 16.1 & China \\
\hline Casale et al. (2016) & & & $x$ & 293 & 21.73 & Italia \\
\hline Çelik y Odaci (2013) & & & $\bar{x}$ & 418 & 20.4 & Turquía \\
\hline Chun (2016) & $x$ & & & 351 & 13.5 & Corea S. \\
\hline Cole y Hooley (2013) & & $x$ & & 163 & 27.3 & EEUU \\
\hline Cudo et al.(2016) & $x$ & & & 260 & 17.5 & Polonia \\
\hline Çuhadar (2012) & & & $x$ & 1235 & 20 & Turquía \\
\hline De Vries et al. (2018) & & $x$ & & 231 & 42.2 & Japón \\
\hline Demirci et al. (2015) & & & $x$ & 319 & 20.5 & Turquía \\
\hline Derbyshire et al. (2013) & & & $\mathrm{X}$ & 2108 & 22.6 & EEUU \\
\hline Durak y Șenol-Durak (2013) & & & $x$ & 448 & 20.53 & Turquía \\
\hline El Asam et al. (2019) & $x$ & & & 1814 & 12.6 & UK \\
\hline Fernández-Villa et al. (2015) & & & $x$ & 2780 & 20.3 & España \\
\hline Huan et al. (2014) & $x$ & & & 681 & 14.5 & Singapur \\
\hline Ioannidis et al. (2018) & & $\bar{x}$ & & 1749 & 29 & Sudáfrica \\
\hline Ivezaj et al. (2017) & & $x$ & & 1000 & 28.5 & EEUU \\
\hline Kelley y Gruber (2013) & & & $x$ & 133 & 19.6 & EEUU \\
\hline Kim et al. (2016) & & $x$ & & 6510 & - & Corea S. \\
\hline Kim y Davis (2009) & & & $x$ & 315 & 22.3 & EEUU \\
\hline Kitazawa et al. (2018) & & & $x$ & 1258 & 19.3 & Japón \\
\hline Laconi et al. (2017) & & $x$ & & 786 & 23.7 & Francia \\
\hline Lee y Stapinski (2012) & & $x$ & & 345 & 29.75 & Australia \\
\hline Lee et al. (2018) & $\mathrm{X}$ & & & 1168 & 14.5 & Corea S. \\
\hline Marín et al. (2018) & $x$ & & & 575 & 14.67 & España \\
\hline Mei et al. (2016) & $x$ & & & 653 & 15.43 & China \\
\hline Odaci (2011) & & & $x$ & 398 & 20.34 & Turquía \\
\hline Odaci (2013) & & & $x$ & 556 & 19.25 & Turquía \\
\hline Odaci y Çelik (2013) & & & $x$ & 424 & 18.69 & Turquía \\
\hline Odaci y Çikrikci (2017a) & & & $x$ & 543 & 18.49 & Turquía \\
\hline Odaci y çikrikci (2017b) & & & $\mathrm{X}$ & 543 & 18.49 & Turquía \\
\hline Odaci y Kalkan (2010) & & & $x$ & 493 & 17.71 & Turquía \\
\hline Özdemir et al. (2014) & & & $\mathrm{X}$ & 648 & 22.46 & Turquía \\
\hline Park y Lee (2017) & $x$ & & & 70696 & 15.10 & Corea S. \\
\hline Park et al. (2014) & $x$ & & & 654 & - & Corea S. \\
\hline Perrella y Caviglia (2017) & $x$ & & & 153 & 15.19 & Italia \\
\hline Quesnel et al. (2018) & & $x$ & & 898 & 27.61 & EEUU \\
\hline Reisoğlu et al. (2013) & & $x$ & & 470 & - & Turquía \\
\hline Rial et al. (2015) & $x$ & & & 1709 & 13.74 & España \\
\hline Romano et al. (2017) & & & $x$ & 60 & 20.74 & UK \\
\hline Sari y Aydin (2014) & & & $\mathrm{X}$ & 525 & 21.8 & Turquía \\
\hline Seabra et al. (2017) & & $x$ & & 1399 & 38.68 & Portugal \\
\hline Senol-Durak y Durak (2011) & & & $\mathrm{X}$ & 521 & 20.32 & Turquía \\
\hline Skues et al. (2016) & & $x$ & & 169 & 26.86 & Australia \\
\hline Tokunaga (2014) & & $x$ & & 179 & 28.6 & EEUU \\
\hline Truzoli et al. (2016) & & & $x$ & 100 & 24.34 & UK \\
\hline Tsitsika et al. (2016) & $x$ & & & 10287 & 15.8 & - \\
\hline Yau et al. (2013) & & $x$ & & 755 & 33.60 & EEUU \\
\hline Zheng et al. (2016) & & $x$ & & 513 & - & China \\
\hline
\end{tabular}

Por otro lado, las variables psicológicas o físicas asociadas al UPI fueron la falta de autocontrol, ansiedad, baja autoestima, baja actividad física y baja autoeficacia académica (tabla 2). La mayoría de los estudios 
centraron su atención en la ansiedad (43.13\%) y autoestima (25.49\%). En menor medida en la actividad física (17.64\%), autocontrol (13.72\%) y autoeficacia académica (9.80\%).

Tabla 2. Variables psicológicas o físicas asociadas al UPI

\begin{tabular}{|c|c|c|c|c|c|}
\hline Referencia & $\begin{array}{c}\text { Auto- } \\
\text { control }\end{array}$ & Ansiedad & $\begin{array}{l}\text { Auto- } \\
\text { estima }\end{array}$ & $\begin{array}{l}\text { Actividad } \\
\text { física }\end{array}$ & $\begin{array}{c}\text { Autoeficacia } \\
\text { académica }\end{array}$ \\
\hline Baloğlu et al. (2018) & & $x$ & & & \\
\hline Bernal-Ruiz et al. (2017) & & $x$ & & & \\
\hline Bozkurt et al. (2013) & & $x$ & & & \\
\hline Cao et al. (2011) & $X$ & & $X$ & $x$ & \\
\hline Casale et al. (2016) & $x$ & & & & \\
\hline Çelik y Odaci (2013) & & & $\bar{x}$ & & \\
\hline Chun (2016) & & & $X$ & & \\
\hline Cole y Hooley (2013) & & $\mathrm{X}$ & & & \\
\hline Cudo et al. (2016) & & & $\mathrm{X}$ & & \\
\hline Çuhadar (2012) & & $\bar{x}$ & & & \\
\hline De Vries et al. (2018) & & $X$ & & & \\
\hline Demirci et al. (2015) & & $x$ & & & \\
\hline Derbyshire et al. (2013) & & $x$ & & & \\
\hline Durak y Şenol-Durak (2013) & & $x$ & & & \\
\hline El Asam et al. (2019) & & & & $\mathrm{X}$ & \\
\hline Fernández-Villa et al. (2015) & & & & $\mathrm{X}$ & \\
\hline Huan et al. (2014) & & $x$ & & & \\
\hline Ioannidis et al. (2018) & & $\mathrm{X}$ & & & \\
\hline Ivezaj et al. (2017) & $x$ & & & & \\
\hline Kelley y Gruber (2013) & & & & $\mathrm{X}$ & \\
\hline Kim et al. (2016) & & $X$ & & & \\
\hline Kim y Davis (2009) & & $\mathrm{X}$ & $\mathrm{X}$ & & \\
\hline Kitazawa et al. (2018) & & $x$ & & & \\
\hline Laconi et al. (2017) & & & $x$ & & \\
\hline Lee y Stapinski (2012) & & $x$ & & & \\
\hline Lee et al. (2018) & & $x$ & & & \\
\hline Marín et al. (2018) & & & & & $X$ \\
\hline Mei et al. (2016) & $x$ & & $x$ & & \\
\hline Odaci (2011) & & & & & $\mathrm{X}$ \\
\hline Odaci (2013) & & & & & $X$ \\
\hline Odaci y Çelik (2013) & & & 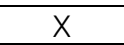 & & \\
\hline Odaci y Çikrikci (2017a) & & $\mathrm{X}$ & & & \\
\hline Odaci y Çikrikci (2017b) & & $x$ & & & \\
\hline Odaci y Kalkan (2010) & & $\mathrm{X}$ & & & \\
\hline Özdemir et al. (2014) & $\mathrm{X}$ & & & & \\
\hline Park y Lee (2017) & & & & $\mathrm{X}$ & \\
\hline Park et al. (2014) & $\mathrm{X}$ & & $x$ & & \\
\hline Perrella y Caviglia (2017) & & & $x$ & & \\
\hline Quesnel et al. (2018) & & & & $\mathrm{X}$ & \\
\hline Reisoğlu et al. (2013) & & & $x$ & & \\
\hline Rial et al. (2015) & & & & & $X$ \\
\hline Romano et al. (2017) & & $x$ & & & \\
\hline Sari y Aydin (2014) & & & & $\mathrm{X}$ & \\
\hline Seabra et al. (2017) & & & $X$ & & \\
\hline Senol-Durak y Durak (2011) & & & $X$ & & \\
\hline Skues et al. (2016) & & & & & $\mathrm{X}$ \\
\hline Tokunaga (2014) & & $x$ & & & \\
\hline Truzoli et al. (2016) & & $X$ & & & \\
\hline Tsitsika et al. (2016) & & & & $\mathrm{X}$ & \\
\hline Yau et al. (2013) & $x$ & & & & \\
\hline Zheng et al. (2016) & & & & $x$ & \\
\hline
\end{tabular}




\subsection{Autocontrol}

La relación del UPI con la falta de autocontrol la establecen distintos estudios (Cao et al., 2011; Casale et al., 2016; Ivezaj et al., 2017; Mei et al., 2016; Özdemir et al., 2014; Park et al., 2014; Yau et al., 2013). Los instrumentos de recogida de datos empleados fueron The Brief Self-Control Scale (BSCS) (70\% de los estudios); Grasmick scale to measure low self-control) (10\%), Difficulties in Emotion Regulation Scale (DERS) (10\%), Self-Control Rating Scale (SCRS) (10\%).

A pesar de la vinculación del UPI con la falta de autocontrol, los datos del metanálisis no especifican diferencias estadísticamente significativas entre el grupo de control y el grupo con UPI $(p=0.97)$ (ver figura 2). La figura del diamante se situó encima de la línea de no efecto, por lo que no se refleja ningún tipo de efecto a favor de un grupo u otro. Aunque de forma particular dos estudios mostraron efecto a favor del grupo de control (Ivezaj et al., 2017; Yau et al., 2013) y otro estudio estuvo a favor del grupo con UPI (Mei et al., 2016). El coeficiente de Cohen no especificó un tamaño del efecto relevante $(d=-0.01$; $95 \% \mathrm{Cl}=-0.63,0.61)$.

Figura 2. Forest plot de Autocontrol

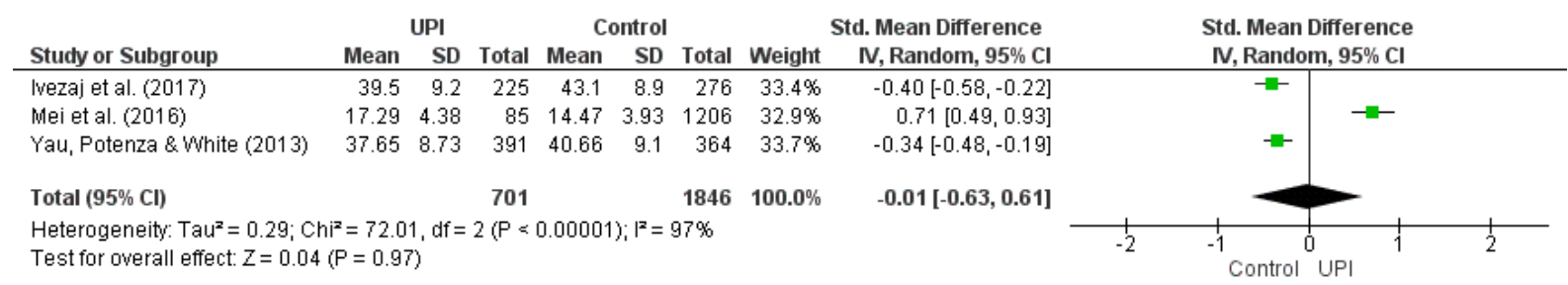

\subsection{Ansiedad}

La ansiedad y su relación con el UPI ha sido estudiada ampliamente (Baloğlu et al., 2018; Bernal-Ruiz et al., 2017; Bozkurt et al., 2013; Cole y Hooley, 2013; Çuhadar, 2012; De Vries et al., 2018; Demirci et al., 2015; Derbyshire et al., 2013; Durak y Şenol-Durak, 2013; Huan et al., 2014; Ioannidis et al., 2018; Kim et al., 2016; Kim y Davis, 2009; Kitazawa et al., 2018; Lee et al., 2018; Lee y Stapinski, 2012; Odaci y Çikrikci, 2017a, 2017b; Odaci y Kalkan, 2010; Romano et al., 2017; Tokunaga, 2014; Truzoli et al., 2016). Los estudios recogen diferentes instrumentos para medir la ansiedad, entre ellos: State-trait Anxiety Inventory (STAI) (25\%); Liebowitz Social Anxiety Scale (LSAS) (20\%); DASS (15\%); K-SADS-PL (10\%), Interactional Anxiousness Scale (IAS) (10\%); Barratt Impulsive Scale (BIS) (5\%), Social Phobia and Anxiety Inventory (SPAI) (5\%), Beck Anxiety Inventory (BAI) (5\%), Sensation-seeking Scale (SS) (5\%).

En relación con el metanálisis, todos los estudios muestran un efecto positivo a favor del grupo con UPI (ver figura 3). Por lo que la ansiedad es mayor en este grupo de sujetos. Por su parte, el tamaño del efecto muestra la significación de los datos $(p=0.00001)$ y la figura del diamante quedó recogida en el extremo derecho a favor del grupo con UPI. El coeficiente de Cohen presentó un tamaño del efecto medio $(d=0.60 ; 95 \% C l=0.37,0.83)$.

Figura 3. Forest plot de Ansiedad

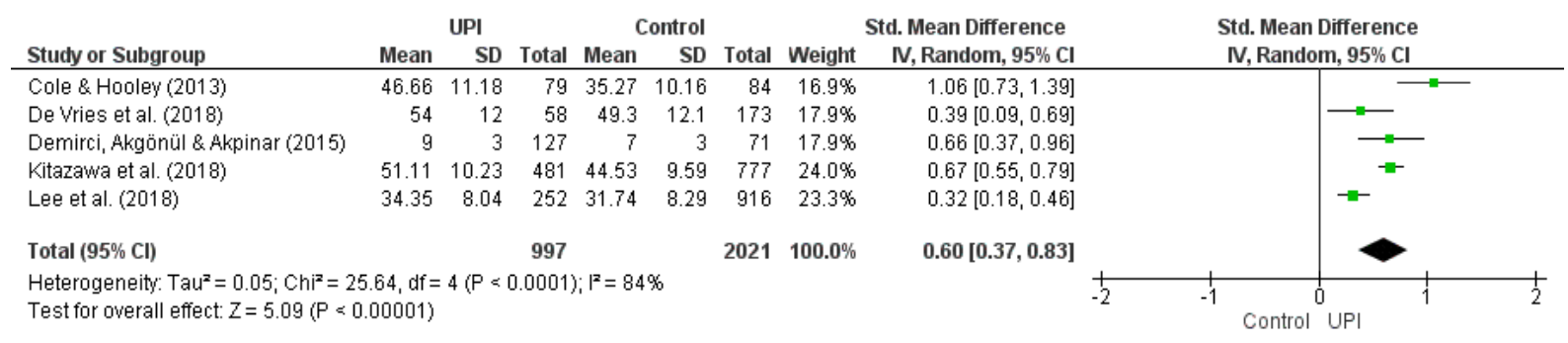




\subsection{Autoestima}

Se recogen distintos estudios que vinculan una baja autoestima con el UPI (Cao et al., 2011; Celik y Odaci, 2013; Chun, 2016; Cudo et al., 2016; Kim y Davis, 2009; Laconi et al., 2017; Mei et al., 2016; Odaci y Çelik, 2013; Park et al., 2014; Perrella y Caviglia, 2017; Reisoğlu et al., 2013; Seabra et al., 2017; Senol-Durak y Durak, 2011). En relación con los instrumentos de recogida de datos, destacan: Rosenberg Self-Esteem Scale (RSES) (60\%); Interpersonal Cognitive Distortions Scale (ICDS) (10\%), Questionnaire of Intrapersonal and Interpersonal Attitudes and Attitudes Towards the World (KNIIS) (10\%), Multidimensional Students' Life Satisfaction Scale (MSLSS) (10\%), Multidimensional Self Concept Scale (MSCS) (10\%).

El metanálisis muestra unas puntuaciones más altas respecto a la autoestima en el grupo control, por lo que la baja autoestima se asocia principalmente al grupo con UPI (figura 4). El tamaño del efecto muestra la significación estadística de los datos $(p=0.010)$. Esto queda reforzado con la ubicación de la figura del diamante a favor del grupo control. El coeficiente de Cohen reflejó un tamaño del efecto adecuado $(d=-$ $0.42 ; 95 \% \mathrm{Cl}=-0.74,-0.10)$.

Figura 4. Forest plot de Autoestima

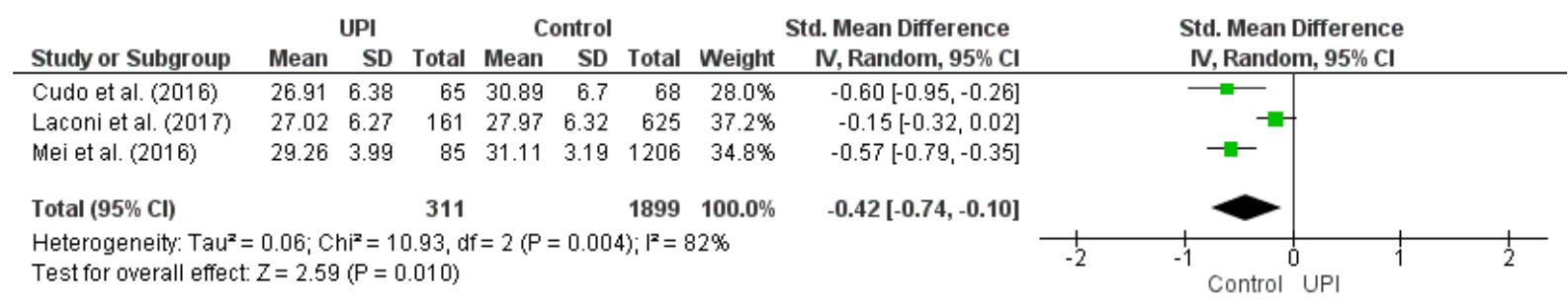

\subsection{Actividad física}

La falta de actividad física también ha sido abordada por distintos trabajos que la asocian con un UPI (Cao et al., 2011; El Asam et al., 2019; Fernández-Villa et al., 2015; Kelley y Gruber, 2013; Park y Lee, 2017; Quesnel et al., 2018; Sari y Aydin, 2014; Tsitsika et al., 2016; Zheng et al., 2016). Principalmente, ha sido medida por el Índice de Masa Corporal (IMC) (35\%) y por distintos instrumentos: Physical and Psychosomatic Health Questionnaire (PPHQ) (15\%), GHQ-12 (15\%), Multidimensional Sub-health Questionnaire of Adolescents (MSQA) (15\%), The SF-36v2 Health Survey (15\%), Exercise Dependence Scale (EDS) (15\%).

A diferencia del resto de meta-análisis, debido a las particularidades de los estudios, se realizó un metanálisis de metodología donde se recoge el total de casos por parte de cada grupo. El forest plot muestra que un único estudio se posiciona a favor del grupo con UPI (Cao et al., 2011), el otro se sitúa en la línea de no efecto (Park y Lee, 2017) (ver figura 5) y la figura del diamante roza la línea de no efecto. Asimismo, el tamaño del efecto no recoge diferencias significativas $(p=0.31 ; \mathrm{OR}=1.92 ; 95 \% \mathrm{Cl}=0.54$, 6.84).

Figura 5. Forest plot de actividad física

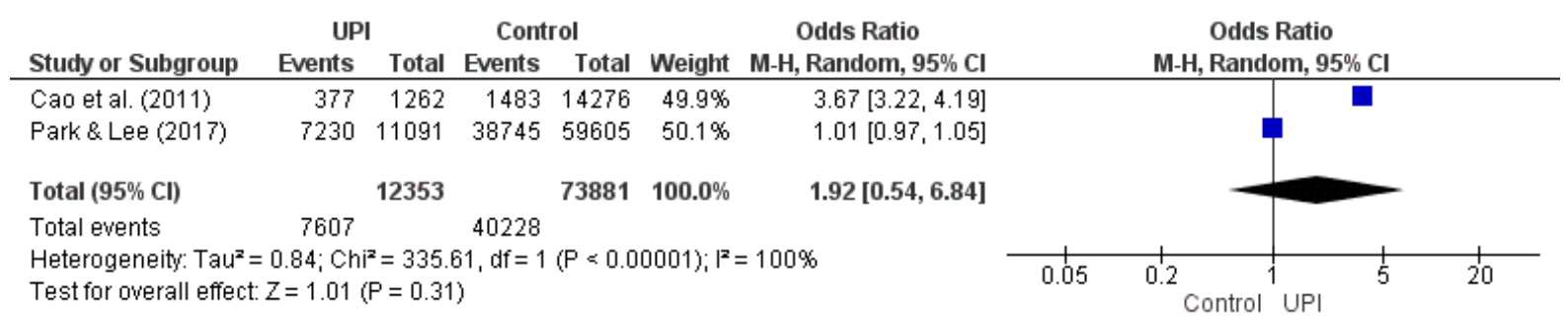




\subsection{Autoeficacia académica}

Este constructo es el único donde no se ha realizado un metanálisis debido a que los datos de los estudios no han permitido realizarlo. No obstante, algunos estudios afirman que el UPI afecta negativamente la autoeficacia académica (Marín et al., 2018; Odaci, 2011, 2013; Rial et al., 2015; Skues et al., 2016). Por su parte, los instrumentos utilizados para la recogida de información han sido la Academic Self-efficacy Scale (ASS) (50\%); Subtest búsqueda de símbolos WAIS-III, Escala de Memoria de Weschler, Test de Aptitudes Diferenciales (DAT), Academic Procrastination Scale (APS), The Distress Tolerance Scale (DTS) (10\%).

\section{Discusión y conclusiones}

La relación del UPI con las variables psicológicas o físicas ha sido abordada en distintos estudios empíricos que abarcan una muestra total de 136760 sujetos. Las variables psicológicas o físicas con mayor incidencia en el UPI son la falta de autocontrol, ansiedad, baja autoestima, baja actividad física y baja autoeficacia académica.

Por su parte, el UPI se ha identificado como uno de los principales riesgos de la sociedad digital (Giménez et al., 2017; Kopecký y Szotkowski, 2017). El abuso de Internet es una de las consecuencias de una sociedad en la que abundan los dispositivos electrónicos. Por lo que es esencial identificar este tipo de consecuencias en la salud de la población y establecer medidas para prevenir el UPI. En esta línea, se ha identificado que la población mayoritaria en riesgo son los estudiantes universitarios (García-Oliva et al., 2017; Ruiz-Palmero et al., 2016). No obstante, aunque en menor medida, los adolescentes y adultos también representan un porcentaje importante en el UPI.

Así pues, esta problemática alcanza un amplio espectro poblacional donde lo casos se dispersan en distintos sectores de la población. A pesar de que los más vulnerables son los estudiantes universitarios y adolescentes, por el tiempo que pasan navegando en la red. A su vez, el UPI alcanza una dimensión global en la que los estudios empíricos se concentran en distintos países de diferentes puntos geográficos (Throuvala et al., 2019).

En concreto, España destaca entre los países de Europa (Díaz-Aguado et al., 2018). Lo cual es significativo si tenemos en cuenta los dos hitos que ocurren en este país respecto a la cantidad de smartphones y la gran conectividad de la población española (Europa Press, 2017; We are social, 2019).

Por otro lado, la revisión de la literatura ha confirmado la asociación del UPI con distintas variables psicológicas (Çam y Nur, 2015; Cao et al., 2011). Entre ellos, los recogidos por Rojas-Jaras et al. (2018), Lei et al. (2018) y Lei et al. (2019): bajo rendimiento académico, baja autoestima, mayor ansiedad y bajo autocontrol.

De manera particular, la ansiedad se alza como el fenómeno más estudiado. Asimismo, la mayor parte de los estudios analizaron la relación entre el UPI como predictor de la ansiedad. Además, se confirma el vínculo del UPI con las variables psicológicas o físicas: autocontrol (Koronczai et al., 2019), autoestima (Zegarra y Cuba, 2017), baja actividad física (Castro et al., 2018) y baja autoeficacia académica (RamosGalarza et al., 2017). Sin embargo, aunque los estudios recogen una vinculación entre el UPI y las variables descritas, el tamaño del efecto global de las investigaciones que estudian el mismo fenómeno recogió que las únicas relaciones estadísticamente significativas eran las de UPI y ansiedad, y UPI y baja autoestima.

De este modo, la evidencia empírica mostró que el UPI es predictor de la ansiedad y de la baja autoestima. El resto de variables a pesar de que se vinculan al UPI no recogen una tasa mayor de prevalencia que sea significativa en la población con UPI.

Por tanto, nos encontramos con un fenómeno que requiere de más investigación para confirmar si el resto de variables psicológicas y físicas pueden ser predictores del UPI. Ya que las relaciones no significativas se han establecido con sólo el tamaño del efecto de tres estudios en el caso de la falta de autocontrol y dos estudios en el caso de la baja actividad física. A lo que se suma el hecho de que para la 
autoeficacia académica no fue posible realizar un metanálisis debido a la falta de datos.

La tasa de prevalencia de UPI aumentará en los próximos años debido al uso incesante de la tecnología. Los estudiantes universitarios son la principal población en riesgo. Se encuentra en manos de los docentes de Educación Superior implementar medidas informativas y formativas en las aulas universitarias para intentar paliar esta problemática. El hecho más preocupante son los efectos negativos que la adicción a Internet presenta en la salud, entre ellos los recogidos en este estudio.

En el desarrollo de este trabajo se ha dado respuesta a los objetivos planteados. De modo que se establece una serie de variables psicológicas o físicas asociadas al UPI y se han identificado las variables con más evidencia empírica y el tamaño relativo del efecto de cada una de ellas, destacando la ansiedad y la baja autoestima como variables significativas.

Entre las implicaciones prácticas de este trabajo se encuentra, por un lado, la elaboración de material informativo sobre las consecuencias del UPI en la población estudiantil, y por otro, los hallazgos del trabajo pueden ayudar a poner el foco de atención en el estudio de la ansiedad y la baja autoestima como principales consecuencias significativas del UPI.

Entre las limitaciones del estudio cabe mencionar la restricción en la búsqueda de información sólo a las bases de datos WOS y Scopus, en futuros estudios sería recomendable ampliar la búsqueda en otras bases de datos; sin embargo, esta decisión garantiza que se ha analizado la literatura con mayor impacto científico. Otra limitación ha sido la confección de los metanálisis que contienen sólo dos artículos, debido a que el resto de estudios no recogieron los datos necesarios para su elaboración. De esta limitación se deriva la recomendación de seguir investigando en futuros trabajos la relación del UPI con las variables psicológicas y físicas detectadas, puesto que la literatura es limitada. Por último, se recoge la limitación del propio sesgo de los investigadores para seleccionar los artículos, el cual se ha intentado paliar con la inclusión de toda la literatura recogida por cada investigador.

Finalmente, la sociedad digital se encuentra con nuevas problemáticas y enfermedades derivadas de un uso constante de la tecnología. Por lo que es crucial enfocarse en la detección de estos problemas que afectan a la salud y a la educación.

\section{Referencias}

Baloğlu, M., Özteke, H. y Kesici, S. (2018). Gender differences in and the relationships between social anxiety and problematic internet use: Canonical analysis. Journal of Medical Internet Research, 20(1), e33. https://doi.org/10.2196/imir.8947

Bernal-Ruiz, C., Rosa-Alcázar, A., González-Calatayud, V. y Rosa-Alcázar, E. (2017). ¿Existe relación entre uso problemático de Internet y las respuestas de ansiedad social, obsesivo-compulsivas y el bienestar psicológico en adolescentes? Anales de Psicología, 33(2), 269-276.

https://doi.org/10.6018/analesps.33.2.265831

Bozkurt, H., Coskun, M., Ayaydin, H., Adak, I. y Zoroglu, S. (2013). Prevalence and patterns of psychiatric disorders in referred adolescents with Internet addiction. Psychiatry and Clinical Neurosciences, 67(5), 352359. https://doi.org/10.1111/pcn.12065

Çam, H. y Nur, N. (2015). A study on the prevalence of internet addiction and its association with psychopathological symptoms and obesity in adolescents. TAF Preventive Medicine Bulletin, 14(3), 181188. https://doi.org/10.5455/pmb.20141016033204

Cao, H., Sun, Y., Wan, Y., Hao, J. y Tao, F. (2011). Problematic internet use in chinese adolescents and its relation to psychosomatic symptoms and life satisfaction. BMC Public Health, 11 (802) 1-8.

https://doi.org/10.1186/1471-2458-11-802 
Casale, S., Caplan, S. y Fioravanti, G. (2016). Positive metacognitions about internet use: The mediating role in the relationship between emotional dysregulation and problematic use. Addictive Behaviors, 59, 84-88. https://doi.org/10.1016/j.addbeh.2016.03.014

Castro, J., Vinaccia, S. y Ballester-Arnal, R. (2018). Ansiedad social, adicción al internet y al cibersexo: su relación con la percepción de salud. Terapia Psicológica, 36(3), 134-143. https://doi.org/10.4067/S0718$\underline{48082018000300134}$

Çelik, C. y Odaci, H. (2013). The relationship between problematic internet use and interpersonal cognitive distortions and life satisfaction in university students. Children and Youth Services Review, 35(3), 505-508. https://doi.org/10.1016/j.childyouth.2013.01.001

Chun, J. (2016). Effects of psychological problems, emotional dysregulation, and self-esteem on problematic internet use among Korean adolescents. Children and Youth Services Review, 68, 187-192. https://doi.org/10.1016/i.childyouth.2016.07.005

Cole, S. y Hooley, J. (2013). Clinical and personality correlates of MMO gaming: Anxiety and absorption in problematic internet use. Social Science Computer Review, 31(4), 424-436.

https://doi.org/10.1177/0894439312475280

Cudo, A., Dobosz, M., Jarząbek-Cudo, A. y Basaj, L. (2016). Problematic internet use and intrapersonal and interpersonal attitudes in adolescents. Advances in Psychiatry and Neurology, 25(3), 159-178.

https://doi.org/10.1016/i.pin.2016.08.005

Çuhadar, C. (2012). Exploration of problematic internet use and social interaction anxiety among Turkish pre-service teachers. Computers \& Education, 59(2), 173-181.

https://doi.org/10.1016/i.compedu.2011.12.029

De Vries, H., Nakamae, T., Fukui, K., Denys, D. y Narumoto, J. (2018). Problematic internet use and psychiatric co-morbidity in a population of Japanese adult psychiatric patients. BMC Psychiatry, 18(9).

https://doi.org/10.1186/s12888-018-1588-z

Demirci, K., Akgönül, M. y Akpinar, A. (2015). Relationship of smartphone use severity with sleep quality, depression, and anxiety in university students. Journal of Behavioral Addictions, 4(2), 85-92.

https://doi.org/10.1556/2006.4.2015.010

Derbyshire, K., Lust, K., Schreiber, L., Odlaug, B., Christenson, G., Golden, D. y Grant, J. (2013). Problematic internet use and associated risks in a college sample. Comprehensive Psychiatry, 54(5), 415-422.

https://doi.org/10.1016/j.comppsych.2012.11.003

Díaz-Aguado, M., Martín-Babarro, J. y Falcón, L. (2018). Problematic internet use, maladaptive future time perspective and school context. Psicothema, 30(2), 195-200.

https://doi.org/10.7334/psicothema2017.282

Durak, M. y Şenol-Durak, E. (2013). Associations of social anxiety and depression with cognitions related to problematic internet use in youths. Education and Science, 38(169), 19-29. https://tinyurl.com/yjozkj9v

El Asam, A., Samara, M. y Terry, P. (2019). Problematic internet use and mental health among british children and adolescents. Addictive Behaviors, 90, 428-436.

https://doi.org/10.1016/i.addbeh.2018.09.007

Europa Press (7 de noviembre de 2017). España, el país con más smartphones por habitante del mundo. https://bit.ly/2WEQnbm 
Fernández-Villa, T., Alguacil, J., Almaraz, A., Cancela, J., Delgado-Rodríguez, M., García-Martín, M., Jiménez-Mejías, E., Llorca, J., Molina, A., Ortíz, R., Valero-Juan, L. y Martín, V. (2015). Uso problemático de internet en estudiantes universitarios: factores asociados y diferencias de género. Adicciones, 27(4), 265275. https://doi.org/10.20882/adicciones.751

García-Oliva, C., Piqueras, J. y Marzo, J. (2017). Uso problemático de internet, el móvil y los videojuegos en una muestra de adolescentes alicantinos. Health and Addictions, 17(2), 189-200.

https://doi.org/10.21134/haaj.v17i2.331

Giménez, A., Luengo, J. y Bartrina, M. (2017). ¿Qué hacen los menores en internet? Usos de las TIC, estrategias de supervisión parental y exposición a riesgos. Electronic Journal of Research in Educational Psychology, 15(43), 533-552. https://doi.org/10.14204/ejrep.43.16123

Hinojo, F., Aznar, I., Romero, J. y Marín, J. (2019). Influencia del aula invertida en el rendimiento académico. Una revisión sistemática. Campus virtuales, 8(1), 9-18.

http://uajournals.com/ojs/index.php/campusvirtuales/article/view/384

Huan, V., Ang, R. y Chye, S. (2014). Loneliness and shyness in adolescent problematic internet users: The role of social anxiety. Child \& Youth Care Forum, 43, 539-551. https://doi.org/10.1007/s10566-014-9252-3

Ioannidis, K., Treder, M., Chamberlain, S., Kiraly, F., Redden, S., Stein, D., Lochner, C. y Grant, J. (2018). Problematic internet use as an age-related multifaceted problem: evidence from a two-site survey. Addictive Behaviors, 81, 157-166. https://doi.org/10.1016/j.addbeh.2018.02.017

Ivezaj, V., Potenza, M., Grillo, C. y White, M. (2017). An exploratory examination of at-risk/problematic internet use and disordered eating in adults. Addictive Behaviors, 64, 301-307.

https://doi.org/10.1016/i.addbeh.2015.11.015

Kelley, K. y Gruber, E. (2013). Problematic internet use and physical health. Journal of Behavioral Addictions, 2(2), 108-112. https://doi.org/10.1556/iba.1.2012.016

Kim, B., Chang, S., Park, J., Jeong, S., Hee, S. y Je, M. (2016). Prevalence, correlates, psychiatric comorbidities, and suicidality in a community population with problematic internet use. Psychiatry Research, 244, 249-256. https://doi.org/10.1016/i.psychres.2016.07.009

Kim, H. y Davis, K. (2009). Toward a comprehensive theory of problematic internet use: evaluating the role of self-esteem, anxiety, flow, and the self-rated importance of internet activities. Computers in Human Behavior, 25(2), 490-500. https://doi.org/10.1016/j.chb.2008.11.001

Kitazawa, M., Yoshimura, M., Murata, M., Sato-Fujimoto, Y., Hitokoto, H., Mimura, M., Tsubota, K. y Kishimoto, T. (2018). Associations between problematic internet use and psychiatric symptoms among university students in Japan. Psychiatry and Clinical Neurosciences, 72(7), 531-539.

https://doi.org/10.1111/pcn.12662

Kopecký, K. y Szotkowski, R. (2017). Cyberbullying, cyber aggression and their impact on the victim -the teacher. Telematics and Informatics, 34(2), 506-517. https://doi.org/10.1016/j.tele.2016.08.014

Koronczai, B., Kökönyei, G., Griffiths, M. y Demetrovics, Z. (2019). The relationship between personality traits, psychopathological symptoms, and problematic internet use: A complex mediation model. Journal of Medical Internet Research, 21(4), e11837. https://doi.org/doi:10.2196/11837

Laconi, S., Vigouroux, M., Lafuente, C. y Chabrol, H. (2017). Problematic internet use, psychopathology, personality, defense and coping. Computers in Human Behavior, 73, 47-54.

https://doi.org/10.1016/i.chb.2017.03.025 
Lee, B. y Stapinski, L. (2012). Seeking safety on the internet: relationship between social anxiety and problematic internet use. Journal of Anxiety Disorders, 26(1), 197-205.

https://doi.org/10.1016/j.janxdis.2011.11.001

Lee, J., Kim, S., Bae, K., Kim, J., Shin, I., Yoon, J. y Kim, S. (2018). Prevalence and risk factors for problematic internet use among rural adolescents in Korea. Asia-Pacific Psychiatry, 10(2), e12310.

https://doi.org/10.1111/appy.12310

Lei, H., Chiu, M. y Li, S. (2019). Subjective well-being and internet overuse: a meta-analysis of mainland Chinese students. Current Psychology, 39, 843-853. https://doi.org/10.1007/s12144-019-00313-x

Lei, H., Li, S., Chiu, M. y Lu, M. (2018). Social support and internet addiction among mainland Chinese teenagers and young adults: a meta-analysis. Computers in Human Behavior, 85, 200-209.

https://doi.org/10.1016/i.chb.2018.03.041

Machimbarrena, J., Calvete, E., Fernández-González, L., Álvarez-Bardón, A., Álvarez-Fernández, L. y González-Cabrera, J. (2018). Internet risks: an overview of victimization in cyberbullying, cyber dating abuse, sexting, online grooming and problematic internet use. International Journal of Environmental Research and Public Health, 15(11), 2471. https://doi.org/10.3390/ijerph15112471

Marín, M., Carballo, J. y Coloma, A. (2018). Rendimiento académico y cognitivo en el uso problemático de internet. Adicciones, 30(2), 101-110. http://dx.doi.org/10.20882/adicciones.844

Martínez, N. y Rodríguez-García, A. (2018). Alfabetización y competencia digital en personas mayores: el caso del aula permanente de formación abierta de la Universidad de Granada (España). Revista Espacios, 39(10), 37. https://www.revistaespacios.com/a18v39n10/a18v39n10p37.pdf

Mei, S., Yau, Y., Chai, J., Guo, J. y Potenza, M. (2016). Problematic internet use, well-being, self-esteem and self-control: data from a high-school survey in China. Addictive Behaviors, 61, 74-79.

https://doi.org/10.1016/j.addbeh.2016.05.009

Moher, D., Liberati, A., Tetzla, J., Altman, D. y The PRISMA Group (2009). Preferred reporting Items for systematic reviews and meta-analyses: the PRISMA statement. PLoS Medicine, 6(7), e1000097.

https://doi.org/10.1371/journal.pmed.1000097

Odaci, H. (2011). Academic self-efficacy and academic procrastination as predictors of problematic internet use in university students. Computers \& Education, 57(1), 1109-1133.

https://doi.org/10.1016/j.compedu.2011.01.005

Odaci, H. (2013). Risk-taking behavior and academic self-efficacy as variables accounting for problematic internet use in adolescent university students. Children and Youth Services Review, 35(1), 183-187.

https://doi.org/10.1016/i.childyouth.2012.09.011

Odaci, H. y Çelik, C. (2013). Who are problematic internet users? An investigation of the correlations between problematic internet use and shyness, loneliness, narcissism, aggression and self-perception. Computers in Human Behavior, 29(6), 2382-2387. https://doi.org/10.1016/j.chb.2013.05.026

Odaci, H. y Çikrikci, Ö. (2017a). An exploration of the associations among internet use, depression, anxiety and stress among youths. Mediterranean Journal of Clinical Psychology, 5(3), 1-16.

https://doi.org/10.6092/2282-1619/2017.5.1635

Odaci, H. y Çikrikci, Ö. (2017b). Differences in problematic internet use based on depression, anxiety, and stress levels. Addicta: The Turkish Journal on Addictions, 4(1), 41-61.

http://dx.doi.org/10.15805/addicta.2017.4.1.0020 
Odaci, H. y Kalkan, M. (2010). Problematic internet use, loneliness and dating anxiety among young adult university students. Computers \& Education, 55(3), 1091-1097.

https://doi.org/10.1016/i.compedu.2010.05.006

Özdemir, Y., Kuzucu, Y. y Ak, S. (2014). Depression, loneliness and internet addiction: How important is low self-control? Computers in Human Behavior, 34, 284-290. https://doi.org/10.1016/j.chb.2014.02.009

Park, S. y Lee, Y. (2017). Associations of body weight perception and weight control behaviors with problematic internet use among Korean adolescents. Psychiatry Research, 251, 275-280.

https://doi.org/10.1016/i.psychres.2017.01.095

Park, S., Kang, M. y Kim, E. (2014). Social relationship on problematic internet use (PIU) among adolescents in South Korea: A moderated mediation model of self-esteem and self-control. Computers in Human Behavior, 38, 349-357. https://doi.org/10.1016/i.chb.2014.06.005

Perrella, R. y Caviglia, G. (2017). Internet addiction, self-esteem, and relational patterns in adolescents. Clinical Neuropsychiatry, 14(1), 82-87. https://www.clinicalneuropsychiatry.org/download/internetaddiction-self-esteem-and-relational-patterns-in-adolescents/

Quesnel, D., Cook, B., Murray, K. y Zamudio, J. (2018). Inspiration or thinspiration: the association among problematic internet use, exercise dependence, and eating disorder risk. International Journal of Mental Health and Addiction, 16(5), 1114-1124. https://doi.org/10.1007/s11469-017-9834-z

Ramos-Galarza, C., Jadán-Guerrero, J., Paredes-Núñez, L., Bolaños-Pasquel, M. y Gómez-García, A. (2017). Procrastinación, adicción al internet y rendimiento académico de estudiantes universitarios ecuatorianos. Estudios Pedagógicos, 43(3), 275-289. http://dx.doi.org/10.4067/S0718-07052017000300016

Reisoğlu, I., Gedik, N. y Göktas, Y. (2013). Relationship between pre-service teachers' levels of self-esteem, emotional intelligence and problematic internet use. Education and Science, 38(170), 150-164.

https://tinyurl.com/yesmohr8

Rial, A., Golpe, S., Gómez, P. y Barreiro, C. (2015). Variables asociadas al uso problemático de internet entre adolescentes. Health and Addictions, 15(1), 25-38. https://doi.org/10.21134/haaj.v15i1.223

Rojas-Jaras, C., Henríquez, F., Sanhueza, F., Núñez, P., Inostroza, E., Solís, A. y Contreras, D. (2018). Adicción a nternet y uso de redes sociales en adolescentes: una revisión. Revista Española de Drogodependencias, 43(4), 39-54. https://www.aesed.com/upload/files/v43n4-2-rrss-r.pdf

Romano, M., Roaro, A., Re, F., Osborne, L., Truzoli, R. y Reed, P. (2017). Problematic internet users' skin conductance and anxiety increase after exposure to the internet. Addictive Behaviors, 75, 70-74.

https://doi.org/10.1016/i.addbeh.2017.07.003

Ruiz-Palmero, J., Sánchez-Rodríguez, J. y Trujillo-Torres, J. M. (2016). Utilización de internet y dependencia a teléfonos móviles en adolescentes. Revista Latinoamericana de Ciencias Sociales, Niñez y Juventud, 14(2), 1357-1369.

http://revistaumanizales.cinde.org.co/rlcsnj/index.php/Revista-Latinoamericana/article/view/2611

Sánchez-Meca, J. (2010). Cómo realizar una revisión sistemática y un meta-análisis. Aula Abierta, 38(2), 5364.

Sari, S. y Aydin, B. (2014). Problematic internet use and body mass index in university students. Eurasian Journal of Educational Research, 54, 135-150. 
Seabra, L., Loureiro, M., Pereira, H., Monteiro, S., Alfonso, R. y Esgalhado, G. (2017). Relationship between internet addiction and self-esteem: cross-cultural study in Portugal and Brazil. Interacting with Computers, 29(5), 767-778. https://doi.org/10.1093/iwc/iwx011

Senol-Durak, E. y Durak, M. (2011). The mediator roles of life satisfaction and self-esteem between the affective components of psychological well-being and the cognitive symptoms of problematic internet use. Social Indicators Research, 103(1), 23-32. https://doi.org/10.1007/s11205-010-9694-4

Skues, J., Williams, B., Oldmeadow, J., y Wise, L. (2016). The effects of boredom, loneliness, and distress tolerance on problem internet use among university students. International Journal of Mental Health and Addiction, 14(2), 167-180. https://doi.org/10.1007/s11469-015-9568-8

Sola, T., Aznar, I., Romero, J. y Rodríguez-García, A. (2019). Eficacia del método flipped classroom en la universidad: Meta-análisis de la producción científica de impacto. Revista Iberoamericana sobre Calidad, Eficacia y Cambio en Educación, 17(1), 25-38. https://doi.org/10.15366/reice2019.17.1.002

Symons, K., Vanwesenbeeck, I., Walrave, M., Van Ouytsel, J. y Ponnet, K. (2019). Parents' concerns over internet use, their engagement in interaction restrictions, and adolescents' behavior on social networking sites. Youth \& Society, 52(8), 1-13. https://doi.org/10.1177/0044118X19834769

Tabachuk, N., Ledovskikh, I., Shulika, N., Kazinets, V. y Polichka, A. (2018). Internet activity and internet addiction: Where is the borderline in developing one's information competency? EURASIA Journal of Mathematics, Science and Technology Education, 14(12), em1640. https://doi.org/10.29333/ejmste/97828

Throuvala, M., Griffiths, M., Rennoldson, M. y Kuss, D. (2019). School-based prevention for adolescent internet addiction: Prevention is the key. A systematic literature review. Current Neuropharmacology, 17(6), 507-525. https://doi.org/10.2174/1570159X16666180813153806

Tokunaga, R. (2014). A unique problem or the manifestation of a preexisting disorder? The mediating role of problematic internet use in the relationships between psychosocial problems and functional impairment. Communication Research, 41(4), 531-560. https://doi.org/10.1177/0093650212450910

Truzoli, R., Osborne, L., Romano, M. y Reed, P. (2016). The relationship between schizotypal personality and internet addiction in university students. Computers in Human Behavior, 63, 19-24.

https://doi.org/10.1016/j.chb.2016.05.010

Tsitsika, A., Andrie, E., Psaltopoulou, T., Tzavara, C., Sergentanis, T., Ntanasis-Stathopoulos, I., Bacopoulou, F., Richardson, C., Chrousos, G. y Tsolia, M. (2016). Association between problematic internet use, sociodemographic variables and obesity among European adolescents. The European Journal of Public Health, 26(4), 617-622. https://doi.org/10.1093/eurpub/ckw028

Varo, C., Murru, A., Salagre, E., Jiménez, E., Solé, B., Montejo, L., Carvalho, A., Stubbs, B., Grande, I., Martínez-Arán, A., Vieta, E. y Reinares, M. (2019). Behavioral addictions in bipolar disorders: A systematic review. European Neuropsychopharmacology, 29(1), 76-97.

https://doi.org/10.1016/i.euroneuro.2018.10.012

We are social (2019). Digital in 2019. España. https://bit.ly/2MNyJyO

Yau, Y., Potenza, M. y White, M. (2013). Problematic internet use, mental health and impulse control in an online survey of adults. Journal of Behavioral Addictions, 2(2), 72-81.

https://doi.org/10.1556/iba.1.2012.015 
Zegarra, C. y Cuba, M. (2017). Frecuencia de adicción a internet y desarrollo de habilidades sociales en adolescentes de una localidad urbana de Lima. Medwave, 17(1), e6857.

https://doi.org/10.5867/medwave.2017.01.6857

Zheng, Y., Wei, D., Li, J., Zhu, T. y Ning, H. (2016). Internet use and its impact on individual physical health. IEEE Access, 4, 5135-5142. https://doi.org/10.1109/ACCESS.2016.2602301 